\title{
Extractive Adversarial Networks: High-Recall Explanations for Identifying Personal Attacks in Social Media Posts
}

\author{
Samuel Carton \\ University of Michigan \\ scartondumich. edu
}

\author{
Qiaozhu Mei \\ University of Michigan \\ qmei aumich . edu
}

\author{
Paul Resnick \\ University of Michigan \\ presnick@umich.edu
}

\begin{abstract}
We introduce an adversarial method for producing high-recall explanations of neural text classifier decisions. Building on an existing architecture for extractive explanations via hard attention, we add an adversarial layer which scans the residual of the attention for remaining predictive signal. Motivated by the important domain of detecting personal attacks in social media comments, we additionally demonstrate the importance of manually setting a semantically appropriate "default" behavior for the model by explicitly manipulating its bias term. We develop a validation set of humanannotated personal attacks to evaluate the impact of these changes.
\end{abstract}

\section{Introduction}

The task of explaining classifier decisions has recently attracted increased attention from the research community. It is important for several reasons, including: 1) The increasing performance gap between simple-and-interpretable models and complex-but-opaque models (which demand more sophisticated explanation techniques); 2) The increasing ubiquity of machine learning in business and government and the concomitant need to understand the decisions of models in highstakes situations; and 3) A rising awareness of the limitations of machine learning and the need for ways to better utilize intrinsically unreliable models (whose weaknesses can potentially be ameliorated by good explanations).

A common way to explain why a model classified an example a certain way is to extract a sparse subset of features that were responsible for the model's decision, sometimes described as a saliency mask or "rationale" in the case of text (Guidotti et al., 2018). This type of local explanation may not completely elucidate why a given example is assigned a given outcome, but it does simplify the relationship by identifying what attributes were considered in the decision.

Existing work on this topic has not explicitly addressed the problem of local feature redundancy. That is, when two features are equally predictive of an outcome, which of them should be included in the saliency mask for that decision? Typical sparsity constraints encourage minimal sufficient masks-unveiling just enough of the example to justify the outcome.

There are domains, however, where it may be important to produce complete explanations rather than minimal explanations. One example is the task of detecting content in online social media that violates a platform's policies. Explanatory models can potentially help human moderators make quicker and more consistent decisions about whether to remove comments (Lakkaraju et al., 2016). However, we propose that truly minimal explanations are liable to give only a partial portrait of why a comment is objectionable, making it harder to render a fair holistic decision. If used to explain to a poster why their post was removed, a minimal explanation can actually be misleading, by implying that some of what was objectionable about their post was benign just because it didn't add marginal signal to the overall classification.

We use an extractive explanatory neural network to identify which social media comments contain personal attacks and which words in those comments are the basis for classifying them as containing personal attacks. We train this model on a large dataset (Wulczyn et al., 2017) of comments labeled for the presence of such attacks, and use the explanatory capacity of the model to identify spans that constitute personal attacks within those comments. We extend the work of (Lei et al., 2016) in using one recurrent neural net (RNN) to produce an explanatory hard-attention rationale 


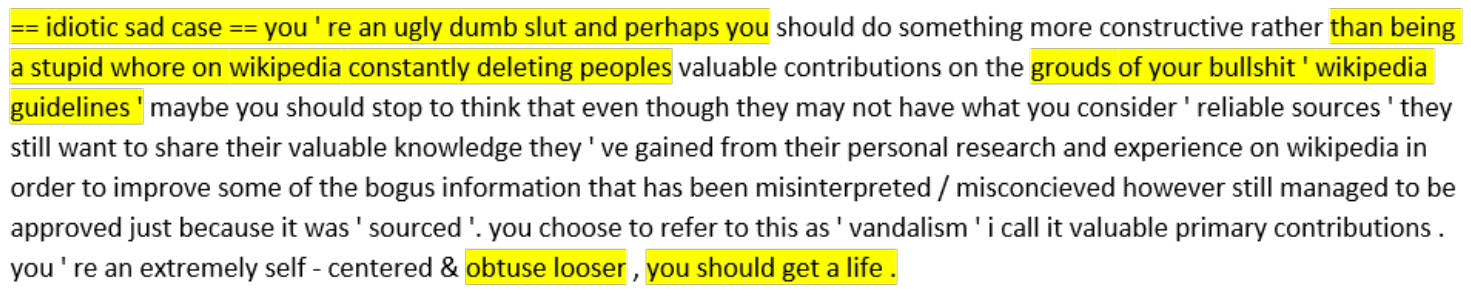

Figure 1: An example of a highly-attacking comment from the test set, rationalized by the model

and a second RNN to make a prediction, the two models trained in an end-to-end fashion.

To produce complete (i.e high-recall) explanations, we add to this existing architecture a second, adversarial predictive layer whose purpose is to try to make predictions based on what is left out of the rationale. We then add a term to the attention layer objective function which encourages it to fool this secondary predictive layer into making poor predictions by including all predictive signal (i.e personal attacks) in the mask that it generates.

We also show that manipulating the model bias term to set a semantically appropriate "default behavior" or "null hypothesis" for the model significantly improves performance. That is, by explicitly choosing what output a zero-information, empty explanation should correspond to, the model is able to learn explanations that correspond more closely with human-generated data.

To summarize, the contributions of this paper are as follows:

- We articulate explanation as an adversarial problem and introduce an adversarial scheme for extraction of complete (high-recall) explanations for text classifier decisions.

- We demonstrate the value of explicitly setting a default output value in such an explanatory model via bias term manipulation.

- We apply explanatory machine learning for the first time to the task of detecting personal attacks in social media comments, and develop a validation dataset for this purpose.

\section{Related work}

\subsection{Online abuse}

Online abuse (of which personal attacks are a major dimension) has recently attracted increased attention as a computational problem. Scholarly work has assessed the prevalence and impact of such abuse (Lenhart et al., 2016; Anderson et al., 2014; Pew, 2016; Anderson et al., 2016), while several initiatives have sought to construct datasets for its study (Wulczyn et al., 2017; Abbott et al., 2016; Kennedy et al., 2017; Napoles et al., 2017; Golbeck et al., 2017).

Naturally, much recent work has gone into the use of machine learning to detect online abuse and its perpetrators (Nobata et al., 2016; Pavlopoulos et al., 2017; Cheng et al., 2015), including a workshop at the most recent ACL conference (Association for Computational Linguistics, 2017). However, the idea of fully-automated moderation by machine learning has attracted criticism as being subject to bias, inaccuracy, manipulation and frustration on the user end (Pavlopoulos et al., 2017; Binns et al., 2017; Blackwell et al., 2018; Hosseini et al., 2017; Adams and Dixon, 2017). We propose interpretable models as one potential solution to some of these problems.

\subsection{Interpretable machine learning}

Major points of division in the interpretability literature include: 1) local vs. global interpretability; 2) post-hoc vs. built-in interpretability; 3) explanation type; 4) input data type; and 5) evaluation metric. Our model is a built-in, feature-based locally interpretable model for text that we evaluate

you were asked nicely and simply responded with dumb insolence. see also this relevant ruling . $\mathrm{i}$ ' $\mathrm{m}$ giving you a three hour block to reflect on this, and expect you to change your signature to something that doesn ' $t$ abuse wikipedia facilities to confuse other editors .

Figure 2: An example of a not-very-attacking example from the test set, rationalized by the model 
relative to a human gold-standard. Guidotti et al. (2018) provides a recent survey of the field.

Recent work on interpretability has focused on local (i.e. instancewise) feature-based explanations. Attention models implicitly produce this type of explanation in the form of attention weights over input features. Lei et al. (2016) utilizes a regularized hard attention mechanism to identify the locally minimum sufficient subset of tokens to make accurate predictions.

Post-hoc methods seek to retroactively probe the behavior of an existing non-explanatory model. These include model-specific gradientbased attribution methods, pioneered by $\mathrm{Si}$ monyan et al. (2013) and reviewed recently by Ancona et al. (2017), which have tended to originate in the image classification domain and transfer to other domains such as text (e.g. Arras et al. (2017)). Conversely, LIME (Ribeiro et al., 2016) is a prominent recent model-agnostic work in this space, building local linear approximations of a model and using the coefficients thereof to explain its behavior. Li et al. (2016) trains a hard attention layer to flip the decisions of an existing model.

While feature-based explanations are the most common approach, other forms have been proposed, including: similarity to learned "prototypes" which represent clusters of items from the training data (Li et al., 2017); high-precision feature interaction rules (Ribeiro et al., 2018); reference to predefined human-friendly concepts (Kim et al., 2017); and generated natural language (Ehsan et al., 2017). Likewise, many evaluation criteria have been proposed. These include fully automated evaluation (Arras et al., 2017); comparison to human gold standards (Lei et al., 2016); and human task performance (Nguyen, 2018). Doshi-Velez and Kim (2017) and Gilpin et al. (2018) both present reviews and taxonomies of evaluation types.

\subsection{Adversarial learning}

Generative Adversarial Networks (Goodfellow et al., 2014) involve the use of a discriminative model to help a generative model match its output to an existing data distribution via an adversarial minimax game. Such models have achieved good results on various generative tasks such as image synthesis (Zhang et al., 2018) and text generation (Yu et al., 2016). Recently, adversarial schemes have begun to be adapted for non-strictly- generative tasks such as fake review detection (Aghakhani et al., 2018), improving the robustness of predictive models to adversarial attacks (Zhao et al., 2017) and image retrieval (Song, 2017).

Ideas similar to the adversarial scheme used in this paper have come not from interpretability but rather from weakly-supervised object localization. Wei et al. (2017) uses a similar scheme to accomplish more complete detection of object shapes in images by iteratively erasing the regions that a predictive model lends the most attention, and forcing it to adjust to the occluded image. Edwards and Storkey (2016) is also similar, using an autoencoder rather than a masking layer to remove identifying information from images.

\section{Model}

The goal of our architecture is to highlight personal attacks in text when such are present, and to highlight little or nothing when there are none, while also performing accurate overall prediction.

These requirements prompt two important edge cases: first, there may be no particular predictive signal in the comment text (i.e. no personal attacks); in a more typical explanatory setting there is always assumed to be some explanation for a decision. Second, there may be redundant signal (i.e. multiple personal attacks), more than is strictly required for accurate prediction, and we assume that it is desirable to identify all of it. We address both of these cases with modifications to the original model architecture.

The model (Figure 3A) is a hard attention architecture which uses one RNN to extract an attention mask of either 0 or 1 for each token, and a different RNN to make a prediction from the attention-masked text (detailed in Figure 3B). Following (Lei et al., 2016), we refer to the maskproducing layer $g$ as the generator, but for clarity we call the predictive layer $f$ the predictor rather than the encoder. Again following previous work, we refer to the output $z$ of the generator as the $r a$ tionale, in that it rationalizes the prediction of the predictor. We also refer to the inverse rationale, defined as $1-z$, as the antirationale.

To this basic two-layer scheme, we add a secondary, adversarial predictor $f_{2}$, which views the text masked by the antirationale rather than the rationale. The secondary predictor's role is to act as an adversarial discriminator-it tries to make accurate predictions on the antirationale, while the 


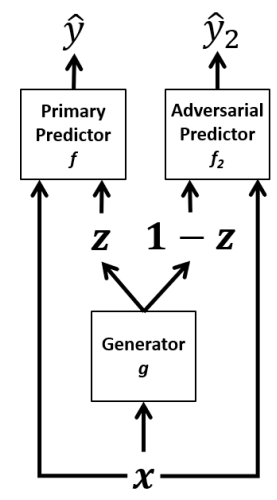

(A)

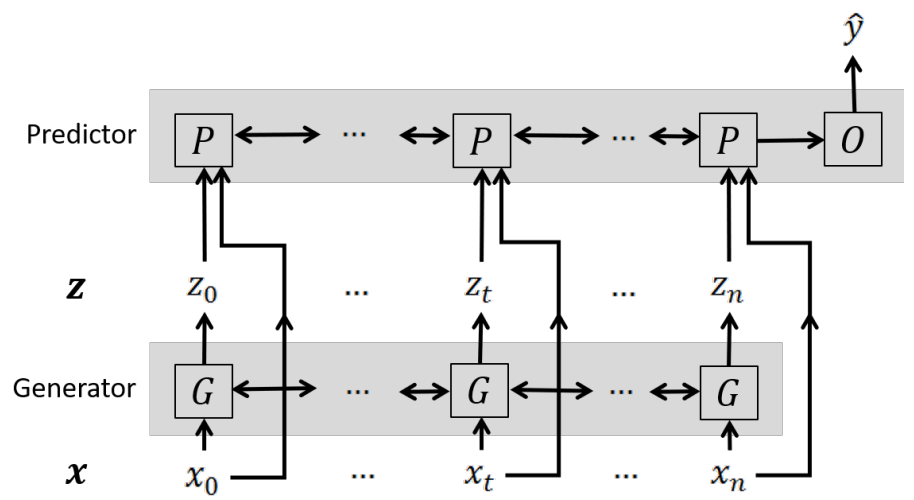

(B)

Figure 3: (A) Overall architecture. Generator and predictors are RNNs; (B) Detail of interaction between generator and one predictor layer. $G$ and $P$ are recurrent units of any kind. $O$ is a sigmoid output layer.

generator tries to prevent it from doing so, which ensures that all predictive signal ends up in the rationale.

\subsection{Primary predictor}

The primary predictor $f$ is an RNN which views the input text masked by the rationale produced by the generator. Its objective is simply to reduce its own squared loss:

$$
\operatorname{cost}_{f}(z, x, y)=[f(x, z)-y]^{2}
$$

\subsubsection{Default behavior via predictor bias term manipulation}

The default behavior of the model is the prediction the predictor makes if the input is entirely masked by the rationale: $f(x, 0)$. When working with a recurrent unit that has no internal bias term, this behavior is entirely determined by the bias term of the final sigmoid output layer, $\sigma(w x+b)$, which with typical random initialization of $b$ results in a default predicted value of roughly 0.5 .

However, this 0.5 default value is not always optimal or semantically appropriate to the predictive task. In the personal attack detection task, if no attacks can be detected, the "natural" default target value for a text should be close to 0 . We show in the experiments that manually setting the output layer bias term $b$ to $\operatorname{logit}(0.05)=-2.94$, so that the default predicted value is 0.05 , improves model performance.

\subsection{Secondary adversarial predictor}

The secondary adversarial predictor is an RNN which views the input text masked by the antira- tionale, defined as 1 minus the rationale $z$. Its purpose is to encourage high-recall explanations by trying to make accurate predictions from the antirationale, while the generator tries to prevent it from doing so.

However, if the adversarial predictor's objective function were simply $\left[f_{2}(x, 1-z)-y\right]^{2}$, it would be able to gain an unfair advantage from the presence of masking in the antirationale. Seeing evidence of "blanked-out" tokens would tell it that personal attacks were present in that comment, giving it strong hint that the target value is close to 1.0 and vice-versa (see figure $4 \mathrm{~A}$ ).

To take away this advantage, the input to the adversarial predictor has to be permuted such that the mask itself is no longer correlated with the target value, while still allowing it to scan the antirationale for residual predictive signal.

Our solution is to replace the masks of half the items in a training batch with the masks of other items in the batch. We order the batch by target value. If item $x_{i}$ is selected for replacement, it gets the mask of item $x_{N-i}$ where $\mathrm{N}$ is the size of the batch. We call this permutation function $c$ :

$$
\begin{aligned}
& c\left(z_{i}\right)=c\left(g\left(x_{i}\right)\right)= \begin{cases}g\left(x_{i}\right) & \text { if } k_{i}=1 \\
g\left(x_{N-i}\right) & \text { if } k_{i}=0\end{cases} \\
& x_{i} \in\left\{x_{0}, \ldots, x_{N}\right\} \quad k_{i} \sim \operatorname{Bernoulli}(0.5)
\end{aligned}
$$

This ensures that low-target-value items get masks associated with high target values and vice-versa, to maximize the dissociation between masks and target values. Figure 4B demonstrates an example of such permutation. This may slow down the learning, since the adversarial predictor 
(A)

\begin{tabular}{|lcccccccc|c|}
\hline \multicolumn{7}{|c|}{ Comment with antirationale } & y \\
\hline moron & , & please & leave & me & alone & . & jerk & . & 1 \\
\hline & you & are & a & real & piece & of & work &. & 1 \\
\hline & why & did & you & revert & my & edits & ? & 0 \\
\hline this & article & is & a & good & piece & of & work &. & 0 \\
\hline don & $\prime$ & t & jerk & me & around & , & thanks &. & 0 \\
\hline
\end{tabular}

(B)

\begin{tabular}{|c|c|c|c|c|c|c|c|c|c|c|}
\hline \multicolumn{9}{|c|}{ Comment with permuted antirationale } & \multirow{2}{*}{\begin{tabular}{|l|}
$y$ \\
1
\end{tabular}} & \multirow{2}{*}{$\frac{\text { Permute? }}{\text { No }}$} \\
\hline moron & , & please & leave & me & alone & . & jerk & . & & \\
\hline & you & are & $a$ & real & piece & of & work & . & 1 & Yes \\
\hline & & why & did & you & revert & my & edits & $?$ & 0 & No \\
\hline this & article & is & $\mathrm{a}$ & good & piece & of & work & . & 0 & No \\
\hline don & ' & $\mathrm{t}$ & jerk & me & around & , & thanks &. & 0 & Yes \\
\hline
\end{tabular}

Figure 4: (A) Fabricated sample batch masked by antirationales. Note the correlation between mask and target; (B) The batch with some antirationales switched with those of other items. The correlation no longer holds.

will sometimes have access to somewhat different features of the input than it will have on the test data, but it should not lead to incorrect learning, since the training data always has the correct label, regardless of the mask.

With $c(1-z)$ as the permuted antirationale resulting from applying this randomization process. The objective for the secondary, adversarial predictor is its predictive accuracy on this permuted antirationale:

$$
\operatorname{cost}_{f_{2}}(z, x, y)=\left[f_{2}(x, c(1-z))-y\right]^{2}
$$

\subsection{Generator}

Given that the two predictors are trying to minimize error on the rationale and (permuted) antirationale respectively, the objective function for the generator is as follows:

$$
\begin{aligned}
& \operatorname{cost}_{g}(z, x, y)= \\
& \quad[f(x, z)-y]^{2} \\
& +\lambda_{1}|| z|| \\
& +\lambda_{1} \lambda_{2} \sum_{t}\left|z_{t}-z_{t-1}\right| \\
& +\lambda_{3}\left[f_{2}(x, 1-z)-f_{2}(x, 0)\right]^{2}
\end{aligned}
$$

Terms 3.1-3.3 are present in the model of Lei et al. Term 3.1 encourages the generator to allow the primary predictor to make accurate predictions, prevents it from obscuring any tokens that would prevent the predictor from doing so. Term 3.2 encourages the generator to produce minimal rationales; obscuring as many tokens as possible. Term 3.3 encourages rationale coherence by punishing the number of transitions in the rationale; it encourages few contiguous phrases rather than many fragments in the rationale.
In theory, these three terms ensure high precision, selecting the minimal (term 3.2) rationale with sufficient signal for accurate prediction (term 3.1), subject to a coherence constraint (term 3.3).

Term 3.4, which is new, ensures recall by encouraging the adversarial predictor's prediction on the antirationale to be similar to the prediction it would make with no information at all (aka the default value). That is, the antirationale should contain no predictive signal. Any personal attacks left out of the rationale would appear in the antirationale, letting the adversarial predictor make a more accurate prediction, which would be penalized by term 3.4 .

\subsection{Extractive Adversarial Network}

In the GAN framework (Goodfellow et al., 2014), a discriminator attempts to accurately classify synthetic examples which a generator is striving to match to the distribution of the true data. In our framework, the adversarial predictor attempts to accurately classify censored examples which the generator is striving to strip of all predictive signal. The discriminator in the GAN framework is trained half on real data, and half on fakes; our adversarial predictor is trained half on correctlymasked items and half on items with permuted masks. Where our framework differs from GAN is instead of generating adversarial examples which are compared to true examples, our architecture extracts a modified example out of an existing example, and so can therefore be described as an Extractive Adversarial Network (EAN).

\subsection{Implementation details}

For comparability with the original algorithm, we use the same recurrent unit (RCNN) and 
REINFORCE-style policy gradient optimization process (Williams, 1992) as Lei et al. (2016) to force the generator outputs to be a discrete 0 or 1. In this framework, the continuous output of the generator on each token is treated as a probability from which the mask is then sampled to produce a discrete value for each token. The gradient across this discontinuity is approximated as:

$$
\begin{aligned}
& \frac{\partial \mathrm{E}_{z \sim g(x)}\left[\operatorname{cost}_{g}(z, x, y)\right]}{\partial \theta_{g}} \\
& =\mathrm{E}_{z \sim g(x)}\left[\operatorname{cost}_{g}(z, x, y) \frac{\partial \log p(z \mid x)}{\partial \theta_{g}}\right]
\end{aligned}
$$

In theory, one would sample $z$ several times from the generator $g$ to produce a good estimate of the gradient. In practice, we find that a single sample per epoch is sufficient. The predictors $f$ and $f_{2}$ are trained as normal, as the error gradient with respect to their parameters is smooth.

We employ a particular hard attention model, but the idea of an adversarial critic is not limited to either hard attention or any particular recurrent unit. In a soft attention setting, our adversarial scheme will actually encourage "harder" attention by encouraging any non-zero attention weight to go to 1.0 (or else the inverse of that weight will leave predictive signal in the anti-explanation).

The attention weights produced by the generator are applied to the predictor at the output rather than the input level. When the recurrent unit $P$ of the predictor operates on a token $x_{t}$ modified by attention weight $z_{t}$, it ingests $x_{t}$ normally, but depending on $z_{t}$ it either produces its own output or forwards that of the previous token:

$$
P\left(x_{t}, z_{t}\right)=z_{t} P_{\text {base }}\left(x_{t}\right) \cdot\left(1-z_{t}\right) P_{\text {base }}\left(x_{t-1}\right)
$$

We investigate a similar range of sparsity hyperparameter values as the original model ${ }^{1}$. The weight on the inverse term only matters relative to the model sparsity, as that term cooperates rather than competing with the predictive accuracy term (because it almost never hurts accuracy to add more to the rationale). Therefore we set $\lambda_{3}$ to 1.0 when we want to include the inverse term.

We use Word2Vec (Mikolov et al., 2013) to create input token word vectors and Adam (Kingma and $\mathrm{Ba}, 2014$ ) for optimization.

\footnotetext{
${ }^{1} \lambda_{1}=[0.0003,0.0006,0.0009,0.0012,0.0015,0.0018$, $0.0021], \lambda_{2}=[0,1,2]$
}

\section{Data}

To train our model of personal attacks, we use the dataset introduced by (Wulczyn et al., 2017), which consists of roughly 100,000 Wikipedia revision comments labeled via crowsourcing for aggression, toxicity and the presence of personal attacks. This dataset includes its own training, development and test set split, which we also use.

To this dataset we add a small validation set of personal attack rationales. 40 undergraduate students used Brat (Stenetorp et al., 2012) to highlight sections of comments that they considered to constitute personal attacks. Comments were sampled in a stratified manner from the development and test sets of the Wulczyn et al. dataset, and each student annotated roughly 150 comments, with each comment viewed by roughly 4 annotators. To calculate gold-standard rationales, we take the majority vote among annotators for each token in each comment. 1089 distinct comments were annotated, split between a development and test set of 549 and 540 examples respectively.

The Krippendorff's alpha on our validation set is 0.53 at the whole-comment level. This value is comparable with that of Wulczyn et al. (2017) (0.45). Agreement at the token level is a lower 0.41 , because this includes tokens which are a matter of preference among annotators, such as articles and adverbs, as well as content tokens.

\section{Experiments}

We show that both modifications to the original algorithm, bias term manipulation and inverse term, increase the tokenwise F1 of the predicted rationales relative to our human-annotated test set. All hyperparameters were tuned to maximize tokenwise F1 on the development set. ${ }^{2}$

\subsection{Baselines}

We generate six baselines for comparison with our variant of the (Lei et al., 2016) architecture. These include the following:

Sigmoid predictor (logistic regression): Bag-ofwords representation with a sigmoid output layer.

RNN predictor: The same sequence model used for the predictor, but with no generator layer.

Mean human performance: The mean tokenwise performance of human annotators measured

\footnotetext{
${ }^{2} \lambda_{1}=0.0006$ for variants without inverse term, $\lambda_{1}=0.0015$ for variant with inverse term, $\lambda_{2}=2$ (Tuned for maximum F1 on original model, then held constant for comparability)
} 


\begin{tabular}{|c|c|c|c|c|c|c|c|c|c|}
\hline \multirow{3}{*}{ Model } & \multicolumn{6}{|c|}{ Rationale } & \multirow{2}{*}{\multicolumn{3}{|c|}{ Prediction }} \\
\hline & \multicolumn{3}{|c|}{ Tokenwise } & \multicolumn{3}{|c|}{ Phrasewise } & & & \\
\hline & F1 & Pr. & Rec. & F1 & Pr. & Rec. & MSE & Acc. & F1 \\
\hline Sigmoid predictor & - & - & - & - & - & - & 0.029 & 0.94 & 0.74 \\
\hline RNN predictor & - & - & - & - & - & - & 0.018 & 0.95 & 0.78 \\
\hline Mean human performance & 0.55 & 0.62 & 0.57 & 0.72 & 0.78 & 0.69 & - & - & - \\
\hline Sigmoid predictor + feature importance & 0.20 & 0.62 & 0.12 & 0.64 & 0.59 & 0.70 & 0.029 & 0.94 & 0.74 \\
\hline RNN predictor + sigmoid generator & 0.29 & 0.22 & 0.45 & 0.31 & 0.19 & 0.92 & 0.038 & 0.91 & 0.70 \\
\hline RNN predictor + LIME & 0.33 & 0.29 & 0.39 & 0.4 & 0.25 & 0.96 & 0.018 & 0.95 & 0.78 \\
\hline Lei2016 & 0.44 & 0.38 & 0.52 & 0.51 & 0.38 & 0.83 & 0.021 & 0.95 & 0.77 \\
\hline Lei2016 + bias & 0.49 & 0.48 & 0.49 & 0.60 & 0.46 & 0.86 & 0.02 & 0.95 & 0.77 \\
\hline Lei2016 + bias + inverse (EAN) & 0.53 & 0.48 & 0.58 & 0.61 & 0.47 & 0.87 & 0.021 & 0.95 & 0.77 \\
\hline
\end{tabular}

Table 1: Rationale performance relative to human annotations. Prediction accuracy is based on a binary threshold of 0.5 . Performance of both Lei2016 model variants is significantly different from the baseline model (McNemar's test, $p<0.05)$

against the majority vote for the comments they annotated (with their vote left out).

Sigmoid predictor + feature importance: Bagof-words representation with sigmoid output layer, with post-hoc feature importance based on model coefficients. Cutoff threshold for features tuned to maximize rationale $\mathrm{F} 1$ on development set.

RNN predictor + sigmoid generator: Rationale mask generated by sigmoid layer applied independently to each input token. Prediction layer is same as predictor.

RNN predictor + LIME: Rationale mask generated by applying LIME (Ribeiro et al., 2016) posthoc to RNN layer predictions. Masking threshold tuned to maximize rationale $\mathrm{F}$.

\subsection{Rationale performance}

In the main experiment, we evaluate model rationales relative to rationales created by human annotators. In our validation dataset, human annotators typically chose to annotate personal attacks at the phrase level; hence in the sentence "Get a job, you hippie s***bag", the majority-vote rationale consists of the entire sentence, where it could arguably consist of the last two or even the last word. Therefore, in addition to tokenwise precision, recall and positive F1, we also report a relaxed "phrasewise" version of these metrics where any time we capture part of a contiguous rationale chunk, that is considered a true positive.

We report results for the original model (i.e. terms 3.1-3.3 in the objective function), the original model with its bias term set for a default value of 0.05 , and the bias-modified model with the additional inverse term (term 3.4). For every model variant, we optimized hyperparameters for tokenwise F1 on the development set. We also report results for the baselines described above.

Table 1 displays the results. The difference in performance between the three baselines that don't use a RNN generator and the three model variants that do demonstrates the importance of context in recognizing personal attacks within text. The relative performance of the three variants of the Lei et al. model show that both modifications, setting the bias term and the addition of the adversarial predictor, lead to marginal improvements in tokenwise F1. The best-performing model approaches average human performance on this metric.

The phrasewise metric is relaxed. It allows a contiguous personal attack sequence to be considered captured if even a single token from the sequence is captured. The results on this metric show that in an absolute sense, $87 \%$ of personal attacks are at least partially captured by the algorithm. The simplest baseline, which produces rationales by thresholding the coefficients of a logistic regression model, does deceptively well on this metric by only identifying attacking words like "jerk" and "a**hole", but its poor tokenwise performance shows that it doesn't mimic human highlighting very well.

\subsection{Original model tokenwise recall}

A perplexing result of the rationale performance comparison is how good the tokenwise recall of the model is without the inverse term. Without it, the model is encouraged to find the minimal rationale which offers good predictive performance. Comments with more than one personal attack 


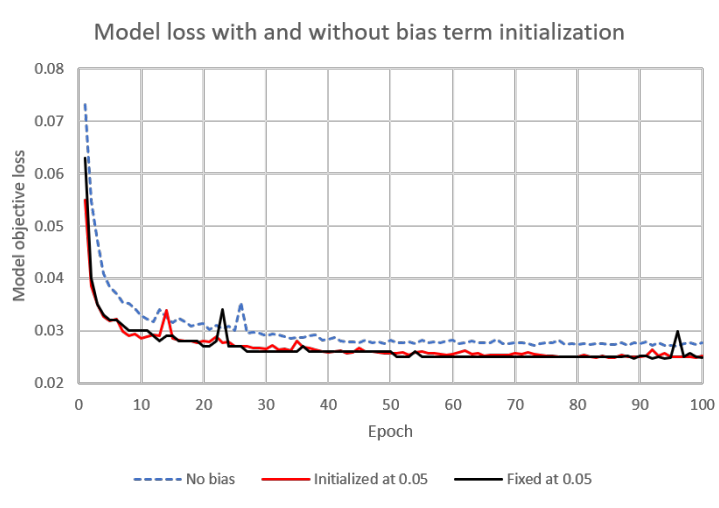

Figure 5: Evolution of model loss over time with and without bias term manipulation

(e.g. Figure 1) constitute $29 \%$ of those with at least one attack and $13 \%$ of all comments in our validation set. For comments like these, the model should in theory only identify one such attack. However, it tends to find more information than needed, leading to a higher-than-expected recall of .52 in the best overall version of this variant.

To explain this behavior, we run a leave-oneout experiment on the original+bias and original+bias+inverse model variants. For each distinct contiguous rationale chunk predicted by each model (when it generates multi-piece rationales), we try removing this chunk from the predicted rationale, running the prediction layer on the reduced rationale, and seeing whether the result lowers the value of the overall objective function.

For the original+bias model variant, we find that performing this reduction improves the value of the objective function $65 \%$ of the time. However, the combined average impact of these reductions on the objective function is to worsen it. What this means is while $65 \%$ of distinct phrases discovered by the generator are unnecessary for accurate prediction, the $35 \%$ of them that are necessary lead to a major decrease in predictive accuracy.

That is, the generator "hedges its bets" with respect to predictive accuracy by including more information in the rationales than it has to, and experiences a better global optimum as a result. This behavior is less prominent with the inclusion of the inverse term, where the percentage of unnecessary rationale phrases falls to $47 \%$.

\subsection{Impact of bias term manipulation}

In theory, the model should learn a good bias term for the predictor layer, and therefore the idea of explicitly initializing or fixing the bias term to match

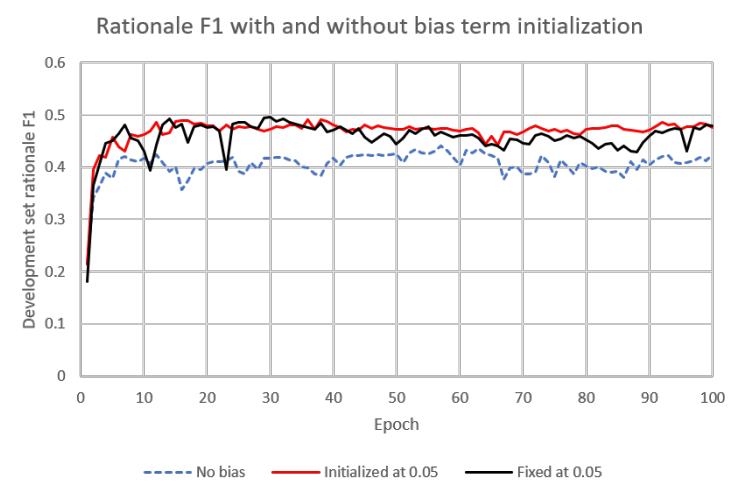

Figure 6: Evolution of development set rationale F1 score over time with and without bias term manipulation

the semantics of the task should not impact model performance or represent much of a contribution.

In practice however, as figures 5 and 6 demonstrate, the initialization of the bias term has a big impact on even the long-term learning behavior of the model. Using the best hyperparameters for the original no-bias, no-inverse-term model, figure 5 shows that either initializing or permanently fixing the predictor bias for a default output value of 0.05 leads to improved model loss with respect to its own objective function. Figure 6 shows a similar pattern for tokenwise F1 score.

\section{Discussion and future work}

One interpretation of the impact of the bias term on model behavior is that an explanation of "why" is really an explanation of "why not"-that is, an explanation is information that distinguishes an item from some alternative hypothesis, and explicitly choosing what this alternative is can improve explanation performance (particularly precision).

Manually setting the model to produce some reasonable default value for an empty rationale makes sense in our setting, but not in domains where there is no default value, such as the beer review dataset of (Lei et al., 2016). A more general approach would be to base explanations on confidence rather than accuracy, where the default value would simply be the mean and variance of the training data, and explanations would consist of tokens that tighten the bounds on the output.

A surprising finding is that the original algorithm often ends up defying its own objective and finds more complete rationales than needed. The leave-one-out experiment described above suggests that the reason for this behavior is that it 


\begin{tabular}{|c|c|c|c|}
\hline & True y & Predicted y & Rationalized comment \\
\hline A) & 0.714 & 0.898 & $\begin{array}{l}==\text { retard }==\mathrm{i} \text { ' ts my flippen opinion anyways and how about get a life intead of reporting peoplego get } \\
\text { laid you stupid gay virgin }\end{array}$ \\
\hline B) & 0.964 & 0.782 & $\begin{array}{l}==\text { laughable censorship }==\mathrm{i} \text { see that a post } \mathrm{i} \text { did in the moon hoax article was ofcourse censored ... fuck } \\
\text { you all admins }\end{array}$ \\
\hline C) & 0.7 & 0.835 & $\begin{array}{l}\text { ::::: what does that have to do with this article ? take whatever preconceived notions you have about me } \\
\text { and shove them up your pussy. }\end{array}$ \\
\hline D) & 0.727 & 0.92 & $\begin{array}{l}\text { i noticed a tweet by you on the topic of cnn ' s don lemon that read, " forget about some measure of } \\
\text { objectivity as an anchor." i was just wondering if you ' re fucking retarded . i mean, you do know you } \\
\text { work for fox news, right ? even if we were to limit the discussion to just you, do you think it would be } \\
\text { difficult for any person with a working cerebral cortext to find one or two hundred clips of you anchoring } \\
\text { on fox and expressing an opinion ? oh, i ' m sorry : not an opinion the opinion of the koch brothers . my } \\
\text { mistake. in conclusion, i ' ' d like to offer you a great big glass of shut - the - fuck - up juice.: }\end{array}$ \\
\hline E) & 0.292 & 0.086 & $\begin{array}{l}==\text { re :== this is a pretty trivial matter, but anyway ... i reverted your " delightful "edit because the } \\
\text { image you added looked tacky . it is hardly the custom for subject userboxes to contain pictures . and it } \\
\text { looks terrible. rest assured, however, that we ' re all just as amazed that you can use latex. wow! }\end{array}$ \\
\hline F) & 0.1 & 0.057 & $\begin{array}{l}\text { i am perfectly within my rights on wikipedia to edit my talk page as i see fit . no user shall edit my page } \\
\text { unless they are adding additional, relevant content. irrelevant content shall be removed. }\end{array}$ \\
\hline G) & 0.3 & 0.057 & '::: an anarchist who supports " intellectual property." amazing .' \\
\hline H) & 0.3 & 0.279 & $\begin{array}{l}==\text { current picture }==\text { can we change it ? maybe one where hes on the middle rope and saying the } \\
\text { second " keeeennnndddeyyyyy " the current one looks a bit retarded " }\end{array}$ \\
\hline
\end{tabular}

Figure 7: Further examples of labeled and rationalized comments. Items E) and G) show that the algorithm struggles with sarcasm.

is how the generator deals with predictive uncertainty, and that it achieves a better global optimum by producing locally suboptimal rationales.

While this "bug" proves useful in our case, it may not generalize. In our setting the adversarial predictor gives a modest improvement in recall; it will produce a larger improvement in settings where the unaltered algorithm is more successful at producing the minimal explanations described by its objective function. Li et al. (2016) finds that a memory network predictor requires less occlusion than an LSTM to flip its predictions, indicating that choice of model can effect completeness of explanations.

In theory, interpretable models can aid human moderaters by pointing them directly at the potentially objectionable content in a comment and giving them a starting point for making their own holistic decision about the comment. However, there are potential pitfalls. Adding explanations as a model output gives the model another way to be wrong-one which humans may be even less able to troubleshoot than simple misclassification. Relatedly, explanations may inspire overconfidence in model predictions. Extensive user testing would clearly be needed before any deployment.

One final concern is the question of whether human-like explanations are really optimal explanations. Are high-recall explanations that mimic human highlighting tendencies really optimal for the types of moderating/self-moderating tasks involved in the domain of personal attacks in on- line social media? Again, this question can only be answered with human subject experimentation, which we plan to approach in future work.

\section{Conclusion}

The main contribution of this paper is to frame explanation as an adversarial problem, thereby addressing explanation recall for the first time that we are aware of. We do so by introducing an adversarial framework (an "extractive adversarial network") for ensuring that redundant predictive signal is not omitted from a model's explanations. We also show that choosing a null hypothesis for the model by setting the model bias term improves explanation precision.

Secondarily, we make a domain-specific contribution by applying interpretable machine learning for the first time to the problem of identifying personal attacks in social media comments, with the hope of developing more transparent semiautomated moderation systems. We show that we approach human performance on a dataset we develop for this purpose.

\section{Acknowledgement}

This material is based upon work supported by the National Science Foundation under grant numbers 1717688, 1633370 and 1620319. We also thank David Jurgens and Yue Wang for their helpful comments and suggestions. 


\section{References}

Robert Abbott, Brian Ecker, Pranav Anand, and Marilyn Walker. 2016. Internet Argument Corpus 2.0: An SQL Schema for Dialogic Social Media and the Corpora to Go With It. In Language Resources and Evaluation Conference (LREC 2016).

CJ Adams and Lucas Dixon. 2017. Better discussions with imperfect models. The False Positive Medium. [Blog post].

Hojjat Aghakhani, Aravind Machiry, Shirin Nilizadeh, Christopher Kruegel, and Giovanni Vigna. 2018. Detecting Deceptive Reviews using Generative Adversarial Networks. arXiv:1805.10364 [cs]. ArXiv: 1805.10364 .

Marco Ancona, Enea Ceolini, Cengiz ztireli, and Markus Gross. 2017. Towards better understanding of gradient-based attribution methods for Deep Neural Networks. arXiv:1711.06104 [cs, stat]. ArXiv: 1711.06104

Ashley A. Anderson, Dominique Brossard, Dietram A. Scheufele, Michael A. Xenos, and Peter Ladwig. 2014. The Nasty Effect: Online Incivility and Risk Perceptions of Emerging Technologies: Crude comments and concern. Journal of Computer-Mediated Communication, 19(3):373-387.

Ashley A. Anderson, Sara K. Yeo, Dominique Brossard, Dietram A. Scheufele, and Michael A. Xenos. 2016. Toxic Talk: How Online Incivility Can Undermine Perceptions of Media. International Journal of Public Opinion Research.

Leila Arras, Grgoire Montavon, Klaus-Robert Mller, and Wojciech Samek. 2017. Explaining Recurrent Neural Network Predictions in Sentiment Analysis. In Proceedings of the 8th Workshop on Computational Approaches to Subjectivity, Sentiment and Social Media Analysis, pages 159-168, Copenhagen, Denmark. Association for Computational Linguistics.

Association for Computational Linguistics. 2017. Proceedings of the 1st Workshop on Abusive Language Online. ACL, Vancouver.

Reuben Binns, Michael Veale, Max Van Kleek, and Nigel Shadbolt. 2017. Like Trainer, Like Bot? Inheritance of Bias in Algorithmic Content Moderation. In Social Informatics, volume 10540, pages 405-415, Cham. Springer.

Lindsay Blackwell, Jill Dimond, Sarita Schoenebeck, and Cliff Lampe. 2018. Classification and Its Consequences for Online Harassment: Design Insights from HeartMob. In Proceedings of the ACM Conference on Computer Supported Cooperative Work (CSCW 2018), pages 1-19.

Justin Cheng, Cristian Danescu-Niculescu-Mizil, and Jure Leskovec. 2015. Antisocial Behavior in Online
Discussion Communities. In Proceedings of the International Conference on Web and Social Media, page 10 .

Finale Doshi-Velez and Been Kim. 2017. Towards A Rigorous Science of Interpretable Machine Learning. arXiv:1702.08608 [cs, stat]. ArXiv: 1702.08608 .

Harrison Edwards and Amos Storkey. 2016. Censoring Representations with an Adversary. In Proceedings of the International Conference on Learning Representations.

Upol Ehsan, Brent Harrison, Larry Chan, and Mark O. Riedl. 2017. Rationalization: A Neural Machine Translation Approach to Generating Natural Language Explanations. arXiv:1702.07826 [cs]. ArXiv: 1702.07826.

Leilani H. Gilpin, David Bau, Ben Z. Yuan, Ayesha Bajwa, Michael Specter, and Lalana Kagal. 2018. Explaining Explanations: An Approach to Evaluating Interpretability of Machine Learning. arXiv:1806.00069 [cs, stat]. ArXiv: 1806.00069.

Jennifer Golbeck, Rajesh Kumar Gnanasekaran, Raja Rajan Gunasekaran, Kelly M. Hoffman, Jenny Hottle, Vichita Jienjitlert, Shivika Khare, Ryan Lau, Marianna J. Martindale, Shalmali Naik, Heather L. Nixon, Zahra Ashktorab, Piyush Ramachandran, Kristine M. Rogers, Lisa Rogers, Meghna Sardana Sarin, Gaurav Shahane, Jayanee Thanki, Priyanka Vengataraman, Zijian Wan, Derek Michael Wu, Rashad O. Banjo, Alexandra Berlinger, Siddharth Bhagwan, Cody Buntain, Paul Cheakalos, Alicia A. Geller, and Quint Gergory. 2017. A Large Labeled Corpus for Online Harassment Research. In Proceedings of the 2017 ACM on Web Science Conference, pages 229-233. ACM Press.

Ian Goodfellow, Jean Pouget-Abadie, Mehdi Mirza, Bing Xu, David Warde-Farley, Sherjil Ozair, Aaron Courville, and Yoshua Bengio. 2014. Generative Adversarial Nets. In Advances in Neural Information Processing Systems, pages 2672-2680.

Riccardo Guidotti, Anna Monreale, Franco Turini, and Dino Pedreschi. 2018. A Survey Of Methods For Explaining Black Box Models. arXiv preprint arXiv:1802.01933.

Hossein Hosseini, Sreeram Kannan, Baosen Zhang, and Radha Poovendran. 2017. Deceiving Googles Perspective API Built for Detecting Toxic Comments. arXiv preprint arXiv:1702.08138.

George Kennedy, Andrew McCollough, Edward Dixon, Alexei Bastidas, John Ryan, Chris Loo, and Saurav Sahay. 2017. Technology Solutions to Combat Online Harassment. In Proceedings of the First Workshop on Abusive Language Online, pages 7377. Association for Computational Linguistics. 
Been Kim, Martin Wattenberg, Justin Gilmer, Carrie Cai, James Wexler, Fernanda Viegas, and Rory Sayres. 2017. Interpretability Beyond Feature Attribution: Quantitative Testing with Concept Activation Vectors (TCAV). arXiv:1711.11279 [stat]. ArXiv: 1711.11279 .

Diederik P. Kingma and Jimmy Ba. 2014. Adam: A Method for Stochastic Optimization. Proceedings of the 3rd International Conference on Learning Representations.

Himabindu Lakkaraju, Stephen H. Bach, and Jure Leskovec. 2016. Interpretable Decision Sets: A Joint Framework for Description and Prediction. In Proceedings of the 22nd ACM SIGKDD International Conference on Knowledge Discovery and Data Mining, pages 1675-1684. ACM Press.

Tao Lei, Regina Barzilay, and Tommi Jaakkola. 2016. Rationalizing Neural Predictions. In Proceedings of the 2016 Conference on Empirical Methods in Natural Language Processing, pages 107-117.

Amanda Lenhart, Michele Ybarra, Kathryn Zickuhr, and Myeshia Price-Feeney. 2016. Online Harassment, Digital Abuse, and Cyberstalking in America. Technical report, Data \& Society Research Institute.

Jiwei Li, Will Monroe, and Dan Jurafsky. 2016. Understanding Neural Networks through Representation Erasure. arXiv preprint arXiv:1612.08220.

Oscar Li, Hao Liu, Chaofan Chen, and Cynthia Rudin. 2017. Deep Learning for Case-Based Reasoning through Prototypes: A Neural Network that Explains Its Predictions. arXiv:1710.04806 [cs, stat]. ArXiv: 1710.04806.

Tomas Mikolov, Ilya Sutskever, Kai Chen, Greg S. Corrado, and Jeff Dean. 2013. Distributed representations of words and phrases and their compositionality. In Advances in neural information processing systems, pages 3111-3119.

Courtney Napoles, Joel Tetreault, Aasish Pappu, Enrica Rosato, and Brian Provenzale. 2017. Finding Good Conversations Online: The Yahoo News Annotated Comments Corpus. In Proceedings of the 11th Linguistic Annotation Workshop.

Dong Nguyen. 2018. Comparing Automatic and Human Evaluation of Local Explanations for Text Classification. In Proceedings of the 2018 Conference of the North American Chapter of the Association for Computational Linguistics: Human Language Technologies, pages 1069-1078.

Chikashi Nobata, Joel Tetreault, Achint Thomas, Yashar Mehdad, and Yi Chang. 2016. Abusive Language Detection in Online User Content. In Proceedings of the 25th International Conference on World Wide Web, pages 145-153.
John Pavlopoulos, Prodromos Malakasiotis, and Ion Androutsopoulos. 2017. Deep Learning for User Comment Moderation. In Proceedings of the First Workshop on Abusive Language Online, pages 2535 .

Pew. 2016. The Political Environment on Social Media. Technical report, Pew Research Center.

Marco Tulio Ribeiro, Sameer Singh, and Carlos Guestrin. 2016. "Why Should I Trust You?": Explaining the Predictions of Any Classifier. In Proceedings of the 22nd ACM SIGKDD International Conference on Knowledge Discovery and Data Mining, pages 1135-1144. ACM.

Marco Tulio Ribeiro, Sameer Singh, and Carlos Guestrin. 2018. Anchors: High Precision ModelAgnostic Explanations. In Proceedings of the AAAI Conference on Artificial Intelligence (AAAI), page 9.

Karen Simonyan, Andrea Vedaldi, and Andrew Zisserman. 2013. Deep Inside Convolutional Networks: Visualising Image Classification Models and Saliency Maps. arXiv:1312.6034 [cs]. ArXiv: 1312.6034

Jingkuan Song. 2017. Binary Generative Adversarial Networks for Image Retrieval. arXiv:1708.04150 [cs]. ArXiv: 1708.04150.

Pontus Stenetorp, Sampo Pyysalo, Goran Topi, Tomoko Ohta, Sophia Ananiadou, and Jun'ichi Tsujii. 2012. BRAT: a web-based tool for NLP-assisted text annotation. In Proceedings of the Demonstrations at the 13th Conference of the European Chapter of the Association for Computational Linguistics, pages 102-107.

Yunchao Wei, Jiashi Feng, Xiaodan Liang, Ming-Ming Cheng, Yao Zhao, and Shuicheng Yan. 2017. Object Region Mining with Adversarial Erasing: A Simple Classification to Semantic Segmentation Approach. arXiv:1703.08448 [cs]. ArXiv: 1703.08448.

Ronald J. Williams. 1992. Simple statistical gradientfollowing algorithms for connectionist reinforcement learning. Machine learning, 8(3-4):229-256.

Ellery Wulczyn, Nithum Thain, and Lucas Dixon. 2017. Ex Machina: Personal Attacks Seen at Scale. In Proceedings of the 26th International Conference on World Wide Web, pages 1391-1399.

Lantao Yu, Weinan Zhang, Jun Wang, and Yong Yu. 2016. SeqGAN: Sequence Generative Adversarial Nets with Policy Gradient. AAAI Conference on Artificial Intelligence.

Han Zhang, Ian Goodfellow, Dimitris Metaxas, and Augustus Odena. 2018. Self-Attention Generative Adversarial Networks. arXiv:1805.08318 [cs, stat]. ArXiv: 1805.08318.

Zhengli Zhao, Dheeru Dua, and Sameer Singh. 2017. Generating Natural Adversarial Examples. arXiv:1710.11342 [cs]. ArXiv: 1710.11342. 